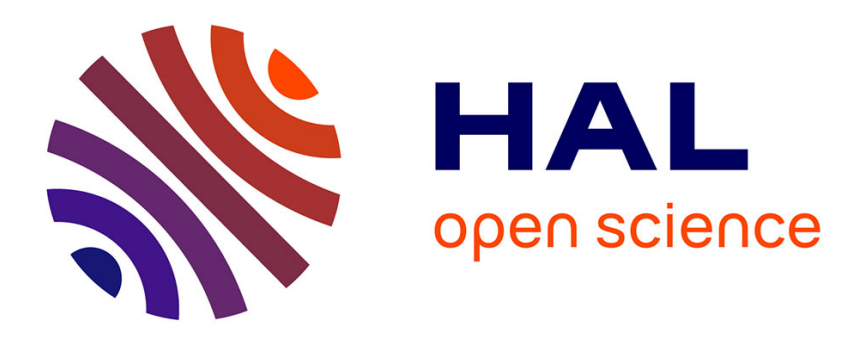

\title{
Être réfugié Rom serbe en Corrèze: saisir sa chance dans un département rural
}

Didier Christophe

\section{To cite this version:}

Didier Christophe. Être réfugié Rom serbe en Corrèze: saisir sa chance dans un département rural. Pour, revue du Groupe Ruralités, Éducation et Politiques, 2017, 230 (16), pp.97-111. 10.3917/pour.230.0097 . hal-01982940

\section{HAL Id: hal-01982940 \\ https://hal.science/hal-01982940}

Submitted on 11 Feb 2020

HAL is a multi-disciplinary open access archive for the deposit and dissemination of scientific research documents, whether they are published or not. The documents may come from teaching and research institutions in France or abroad, or from public or private research centers.
L'archive ouverte pluridisciplinaire HAL, est destinée au dépôt et à la diffusion de documents scientifiques de niveau recherche, publiés ou non, émanant des établissements d'enseignement et de recherche français ou étrangers, des laboratoires publics ou privés. 
CHRISTOPHE Didier, « Être réfugié Rom serbe en Corrèze : saisir sa chance dans un département rural », Pour, 2016/2 (N²30), p. 97-111. DOI : 10.3917/pour.230.0097. URL : https://www.cairn.info/revue-pour-2016-2-page-97.htm

[Version auteur]

\section{Être réfugié Rom serbe en Corrèze : saisir sa chance dans un département rural}

Didier CHRISTOPHE, Formateur en éducation socioculturelle à l'ENSFEA

Chaban est arrivé en Corrèze avec sa famille, il y a huit ans. Dans ce département rural, il trouve des conditions favorables à son intégration.

Chaban Tatari est Ashkali ${ }^{1}$ par son père, tandis que sa mère est Rom ; tout sont originaires du. S'il a un titre de séjour qui lui permet de travailler, il n'est pas reconnu par la Serbie où il est né. N'ayant pas encore rempli les conditions requises, il ne peut pas demander la nationalité française, notamment du fait qu'il n'a pas encore cumulé cinq ans d'emploi en France, et que les autorités serbes lui refusent la délivrance de son certificat de naissance. Il a pu commencer à s'intégrer en travaillant dans des entreprises rurales, après une apprentissage réussi dans la petite ville de Tulle.

Nous avons souhaité nous interroger ici sur les facilités d'insertion qu'offre un département rural pour ces migrants. Mais dans les échanges, le parcours traumatique des peuples nomades croise sans cesse nos questionnements d'Occidentaux paisibles et confortablement installés, alors que l'une des raisons qui ont poussé les Tatari et certains de leurs amis à quitter la Serbie a été l'incendie volontaire du bidonville où ils vivaient.

La famille Tatari est originaire du Kosovo, un des états nés de l'explosion de la Yougoslavie. Les différentes communautés tziganes, composées principalement des peuples Rom, Ashkali et Gorani, comptaient 100.000 personnes au Kosovo au début des années 1990. Avec les Serbes et les Juifs Kosovars, elles ont fait partie des nombreuses

1 Les Ashkali, qui prétendent être originaires d'Égypte, sont islamisés depuis la domination turque sur les Balkans. Ils sont un peuple de 20.000 personnes, albanophone, éclaté sur différents pays, principalement Kosovo (pour près des trois-quart d'entre eux), Albanie, Serbie, Monténégro et Macédoine. II s'estiment être un peuple différent des Roms. Certains groupes se dénomment en référence à leur origine supposée : Egyptiens. Au Kosovo en 2009, alors que le taux de chômage national atteint $45 \%$, les Ashkali sont à $98 \%$ chômeurs, et vivent d'expédients, friperie, recyclage, jardinage, etc. Cf. Jacqueline Bhabha et Al. (2014), « Post-war Kosovo and its policies towards the Roma, Ashkali, and Egyptian communities " (rapport), Harvard School of Public Health, Boston; et Muhamet Arifi (2009), « Notes made from the Ashkali and Egyptian communities for the shadow report on the Implementation of the Framework Convention for the Protection of Nationa Minorities in Kosovo » (rapport), publié par Boris Tsilevich sur le site cilevics.eu/minelres/ (Minority Electronic Resources). 
cohortes par lesquelles 230.000 membres de ces minorités quittèrent leur pays sous les bombardements pendant la guerre. Vingt-cinq ans plus tard, ils ne seraient que 30.000 tziganes à être restés au pays et à peine plus de 6.000 à y être revenus. Le Comité de Surveillance de I'OTAN au Kosovo indiquait en juillet 2003 que depuis juin 1999, en quatre ans, au moins 14.000 maisons avaient été détruites et non reconstruites, appartenant à des Roms et autres communautés (dont de nombreuses familles serbes), et que plus de 3.000 Roms ont trouvé la mort dans ces violences ethniques ${ }^{2}$. Les Tatari, comme nombre de leurs compatriotes, ont été contraints de partir vers des pays voisins qui ne souhaitaient pas davantage leur présence, comme la Serbie (où ils sont arrivés durant la guerre et qui fut leur nouveau pays pendant une quinzaine d'années) ou l'Albanie. Après diverses tentatives de fuite, ces personnes, qui étaient sédentarisées depuis plusieurs générations au Kosovo, ont dû vivre l'éclatement de leur famille, répartie maintenant entre la Serbie, l'Allemagne et la France.

Chaban Tatari a donc échoué à Tulle par les hasards et les aléas des migrations, avec des frères, belle-sœur, neveu, et deux familles amies. II a alors fallu trouver les moyens de s'insérer, par la scolarité et l'emploi, dans les bourgs voisins, Égletons, Uzerche, Argentat, ou encore Treignac où certains ont suivi quelques années de scolarité, vivent et travaillent désormais. Chaban vit maintenant avec une amie française avec laquelle il a deux enfants. Ses parents ont quant à eux aboutit dans la banlieue de Berlin.

Un long entretien nous permet d'appréhender dix clés de compréhension des enchaînements factuels vécus et des mécanismes d'intégration.

\section{Clé $n^{\circ} 1$ : le parcours semé d'embûches d'immigrés venus de Serbie, entrés dans I'UE par la Hongrie.}

Arrivé vers Subovica, le chauffeur du camion qu'ils ont payé d'avance pour le émigration leur demande de passer la frontière à pied par la forêt en leur disant qu'il les reprendra en charge au village suivant mais les abandonne. Ils ne tardent pas à être repérés par la police hongroise

D. C. - En Hongrie...

2 Cf. Marie-Pierre Lahaye (2003), « Un témoignage direct sur le sort des minorités au Kosovo », in B. I. Balkans-Infos, Paris, $N^{\circ} 79$, et repris par le site du Comité de Surveillance de I'OTAN au Kosovo, csotan.org. 
C. T. - Voila, en Hongrie. Après, là-bas on est restés, si je me souviens bien, six mois, on a donné les empreintes et tout ça, et puis on a attendu la réponse, quoi. Du coup, c'était en négatif. Et puis et puis, ils voulaient nous renvoyer en Serbie, et nous on voulait pas, parce qu'on avait des problèmes là-bas [...]. Et après, heu, on est arrivés en Hongrie, on a demandé asile, c'était refusé, et après ils voulaient nous expulser en Serbie Et puis mon frère... on avait acheté deux voitures, on a demandé à la famille qu'ils nous aident, d'Allemagne, on a acheté deux voitures là-bas, et puis on a monté vingt-cinq personnes, sur deux voitures, du coup on est partis, on a pris la route pour venir en France, c'était pour, si je me souviens bien, c'était pour Nice, un truc comme ça, non... ouais, je pense c'était à Nice... je sais pas, ça fait longtemps. Après y a une voiture qui a tombé en panne, du coup qu'est-ce qu'on a fait, on a mis vingt-cinq personnes dans une voiture, et puis, et puis, on a continué, on a continué comme ça, parce que vu que la voiture elle a cassée en Italie, à Torino, voila. De Torino jusqu'à Dijon, à vingt-cinq personnes dans une voiture, c'était une Mercedes, si je me souviens bien, [...] voilà quoi, et du coup, la voiture aussi elle est tombée en panne au milieu de l'autoroute, et après il y avait un très très grand bouchon. [...] Vu que on était vingt-cinq dans la voiture, et puis les gens quand ils ont vu qu'on sortait tout le monde dans une voiture, ils ont appelé les flics, et la gendarmerie ils sont venus, et après, ils nous ont envoyé là-bas, ils nous ont envoyé à... comment il s'appelle...?

- Là, c'était en allant sur Dijon...

- Oui, voila, à la gendarmerie, quoi. Voilà. Et du coup là-bas on a demandé asile aussi, on devait, normalement rester, je pense, dans quinze jours normalement on devait avoir une réponse, ils ont même pas attendu le quinzième jour, quand est arrivé le quatorzième jour, par exemple, demain on devait avoir la réponse, et par exemple, cette nuit-là, ils sont arrivés, cent gendarmes un truc comme ça, y avait partout les flics, du coup ils nous ont emmené en Hongrie, directement à l'aéroport et ils nous ont fait expulser. Après, quand est arrivés là-bas, on est restés... c'était... une semaine.

- Ils vous ont renvoyé en Hongrie...

- En Hongrie, c'est bien ça. C'est ça. Et après, quand on est arrivés, là-bas... et après làbas j'étais majeur, ils m'ont séparé de ma famille. Et on a resté une semaine dans un camp où c'est tout fermé comme une prison, après, après une semaine ou vingt jours, je m'en rappelle plus, ils te mettent dans un camp libre, tu peux sortir en ville, tu peux sortir où tu veux, voila. Et là on a demandé encore à acheter une voiture, on a demandé, ma famille, nous aider pour acheter une voiture, on avait acheté une Suzuki Liana. Y avait deux frères, deux frères qui sont restés là-bas, ils pouvaient pas nous suivre, du coup ils 
ont demandé pour revenir. Mais nous qu'est-ce qu'on a fait, on a fuit encore une fois, de Hongrie, parce qu'on voulait pas revenir là-bas, en Serbie. Du coup, on a monté quinze personnes dans une voiture, on est venu, direction Lille. Ouais. C'était ça, Lille. Et après... après, après, mon frère il s'est trompé de péage, je me rappelle pas où c'était où, et du coup, au lieu de partir dans un péage pour pièces, il est parti à la carte, on savait pas comment ça marche.

- Oui.

- Y avait les gendarmes en face, dans une aire de repos, et du coup on nous a arrêtés là, et depuis, depuis là, nous a envoyé à Brive [Brive-la-Gaillarde], on a été... une semaine à Brive. Ils nous ont dit dans cinq jours, normalement, vous serez... vous serez, normalement... expulsés, vous allez en Hongrie, ou Serbie, je m'en rappelle plus. Voilà. Et...nous, on a dit Ok, tout. On a attendu, on a attendu, comme ça est arrivé une semaine, en fait, y avait un bus, minibus, qui est venu nous chercher, qui nous a amené directement à... là... à Tulle, sur le HLM Pièce-Verdier. Et là-bas, quand on est arrivés, on avait deux appartements, vu qu'on était nombreux, et du coup y avait à manger, ils ont fait tout, déjà préparé, les caisses et tout ça, y avait des nouilles, des trucs, y avait des tas de trucs, et nous on a pas compris pourquoi... Normalement, on devait être, on devait être déjà expulsés. Et du coup, depuis là, et on est toujours là, et puis y avait les voisins qui nous a aidés.

- Mais alors vous êtes arrivés, donc ça fait... ça fait sept ans je crois...

- Non, ça fait, je pense, ça fait huit ans. On est arrivés en 2009. Juin, je pense, juin 2009. Un truc comme ça, je pense.

- Donc quand vous êtes arrivés ici, donc toi tu venais d'avoir dix-huit ans quelques mois ou quelques semaines avant.

- C'est ça.

- Vous êtes arrivés ici, et en arrivant Pièce Verdier... vous, vous avez été logés... je ne rappelle pas, vous aviez deux appartements, pour vous quinze ?

- Oui, c'est ça. Et un avec l'étage. Parce qu'il y en avait un, où mon frère, où il habitait, il y avait trois chambre, je pense, voilà, oui, c'était ça, trois chambres, salle de bain, salon, un très grand salon, cuisine. Après, dans l'autre côté, y avait un grand salon, y avait une chambre, cuisine, et en haut il y avait trois chambres, parce que...

- Oui, un duplex.

- Oui, parce qu'il y avait un escalier à l'intérieur.

\section{Clé $n^{\circ} 2$ : les conséquences de la purification ethnique.}


- Et tu as, ici en France, tu as rencontré d'autres gens qui ont un peu le même parcours, c'est-à-dire Roms venant de l'ex-Yougoslavie, comme ça ?

- Oui j'en connais un, j'en connais un, qu'il est venu avec la famille, parce que....

- Oui, mais... venu avec sa famille...

- Avec sa famille à lui, quoi.

- Oui, oui.

- Voilà. Venu avec sa famille. D'ailleurs ça fait combien de temps ? Ça a fait à peu près quatre ou cinq ans qu'il est là, et du coup, du coup, du coup, il avait des problèmes là-bas avec les Albanais, parce que là-bas aussi, c'est en Kosovo, c'est pareil, comme les Serbes, si... par exemple, si t'as pas la peau blanche, ils vont pas t'accepter, parce que les Albanais ils sont blancs, et du coup, si t'as la couleur, peau mate, ils vont pas t'accepter, parce que, en fait, comment dire, ils jouent à... comment dire... taureau, quoi, comme un peu taureau. Si par exemple les Albanais ils disent «Wah! Vous avez rien à foutre là, allez-y en Serbie »; qu'on va en Serbie, la Serbie, ils disent "Qu'est-ce que vous faites là ? Allez-y en Kosovo ». Nous, en fait nous on est entre les deux feux.

- Oui.

- Voilà. Et du coup, on n'a pas où on va aller, on n'a pas de pays, on a rien, les gens, ils nous rejettent. Quoi, ils jouent avec nous comme si on était un ballon, quoi, et voila quoi ; et pas... Le monsieur, là, qu'est venu là, c'est un Rom, et du coup, il avait des problèmes, ils lui ont massacré sa famille, son père, j'pense, et un truc comme ça, quoi.

- Au Kosovo?

- Au Kosovo, voila, pendant la guerre, et du coup maintenant, il a des problèmes un peu... la psychiatrie, je pense, un truc comme ça, et il en... parce qu'il tenait une boucherie avec son père, mais les Albanais, ils lui ont foutu le feu et tout ça, ils ont tué son père, j'pense, et j'sais pas quoi, de la famille, j'sais pas quoi d'autre, du coup, il est parti du Kosovo parce qu'il s'est fait menacer. Du coup il est venu à Tulle. Après, j'pense pas que j'en ai connu d'autres. D'autres gens de... de là-bas. Après, plutôt Marocains, Turcs, comme ça, quoi. Voilà.

\section{Clé $n^{\circ} 3$ : l'insertion par le sport.}

- Vous êtes plusieurs à avoir fait du foot, en arrivant.

- Oui, oui. Et on était... À l'époque, y avait que moi.

- Y avait que toi, au début? 
Voilà, y avait que moi, parce que mes petits cousins, c'était trop petit pour le faire. C'était pas... le problème, c'était pas trop petit, c'est le problème que... heu... J'sais pas, chez nous, ce n'est pas vraiment l'habitude de laisser les enfants, quand ils sont petits, aller tout seuls par exemple quelque part, ou... enfin, tout seuls, voila. Et du coup, j'y ai été un peu tout seul, et après, à chaque fois, quand j'allais faire des entraînements, je les prenais avec mois. Et voilà, c'est comme ça que ça a commencé un petit peu, et après, une fois qu'ils ont été grands, qu'on a commencé un peu de parler la langue...

Tu les as rencontrés comment, les... le club de foot?

Avec des amis, c'est avec des amis. Je...

Que tu avais rencontrés à Pièce-Verdier?

Voilà, c'est ça. Je... Voilà, c'est ça. J'allais avec une amie, une fois, au foot-salle, et c'est là que j'ai commencé de connaître des gens qui m'ont dit « Vas-y, viens jouer au foot-salle avec nous et tout, ouais ». Je commence à jouer, après, ils ont vu que je joue bien, et tout ça, ils m'ont dit « Ouais, mais il faut que tu ailles dans un club, et tout ça ». Après, mois j'ai dit que je connais pas les endroits. Après ils m'ont amené à... mon premier club, c'était à Saint-Martial-de-Gimel, c'était en division... c'était tout mon premier club, c'était en division... heu, 3 ou 4, je me rappelle plus. Et après, j'avais des problèmes papiers, à l'époque, parce que quand on avait signé les parrainages et tout ça...

Oui... vous avez été parrainés... par des élus et des responsables associatifs.

Voilà, y avait le président de mon club qui devait venir pour le parrainage, mais malheureusement il était pas là, il était absent, raison personnelle, et du coup, il a envoyé sa vice-présidente du club. Et sa vice-présidente du club, elle travaille à la préfecture de la Corrèze. Et du coup, elle avait pas droit à signer un truc. [...] De toutes façons moi je l'ai dit, moi je préfère partir du club que par exemple la dame qui perd son boulot à cause de moi. Je l'ai dit, moi je vais partir, voilà, j'ai pas envie de créer des problèmes. Je suis pas venu pour créer des problèmes. Je suis juste venu... avoir la tête tranquille ; et pas créer plus de problèmes. Et puis, et puis et puis après, on est allés faire des tournois avec mes copains du club, mais j'étais pas dans le club à eux, mais on a fait un tournoi sixte. Du coup, c'est libre et tout, on a pu faire, et du coup j'ai rencontré d'autres gars, ils m'ont dit «Ouais, il faut que tu viennes dans notre club, et tout... » Voilà. Et c'était à Tulle, Tulle Football Corrèze [TFC]. Et c'est là que j'ai commencé jouer. À l'époque où j'ai commencé, j'ai commencé en une bonne division, c'était en PL [promotion de ligue], promotion d'honneur, heu, et après c'est... après je commençais un peu après, un peu à jouer dans la $C$ [l'équipe $C$ du TFC], parce qu'il y avait plusieurs équipes...

Oui... 
Voilà, y avait, c'était pour dépanner, certains endroits, voilà, quoi. Et puis, et puis après je suis retourné encore à Saint-Martial, parce qu'ils ont fait pas mal de choses pour moi, ils m'ont aidé, moi je voulais les aider. Voilà, quoi. Et puis et puis, maintenant, maintenant je change encore d'autre club, je suis avec l'équipe du Maumont [à Saint-Mexant en Corrèze, l'Olympique du Maumont a deux équipes en 3ème et 4ème divisions].

\section{Clé $n^{\circ} 4$ : les voisins, les aidants.}

- Et quand vous êtes arrivés, et que vous aviez cet appui, ce soutien, en arrivant ici, au moment où il y avait les risques d'expulsion, tu expliquerais ça comment, le fait que les gens, Alexandra, mais pas seulement... qu'il y a eu cet appui ?

- Mais, après... Y avait des voisins, après. $Y$ avait des voisins, que c'étaient prêt à faire n'importe quoi pour nous. C'étaient prêt de bloquer toutes les routes, que la police qui vient pas pour nous faire expulser.

- Tu expliques comment? Tu sais comment, tu sais un peu pourquoi les gens ont réagi comme ça?

- Parce que, je pense, moi, parce que, comment dire, sûrement parce que, je sais pas... Parce que... je sais pas. Je sais pas comment expliquer ça.

- Parce que c'est pas la situation classique, quand même ! Alors...

- Peut-être parce qu'ils ont, certains, parce que en fait certains voisins qu'on avait, c'était des français, mais c'était d'origine... étrangers. Certains.

- Oui.

- Et sûrement, ils connaissent déjà un peu leurs vies de leurs grands-parents et tout ça, et à mon avis, à mon avis, ça doit être ça, mais y avait des français aussi, hein, y avait des français qui nous ont défendus. Parce qu'on a expliqué, on a expliqué notre cas, aussi, comment, qu'est-ce qui... carrément, là, notre histoire.

- Oui.

- Et du coup les gens, ils ont dit... voilà, quoi. On va, ou va les aider. Après, ils ont fait des... des pétitions, et tout, voilà, quoi. Y avait je sais pas combien de pétitions.

- Tu penses que c'est plutôt de la solidarité, tu dirais que c'est de la solidarité, de la résistance... que y a un côté plutôt humain, plutôt politique ?

- Mais, je pense, plutôt humain. Ouais, je pense que plutôt humain, parce que y avait rien, y a rien à voir avec la politique. Je pense que plutôt humain, parce que les gens ils étaient sympas, et ils voulaient aider, quoi. Parce que, mais moi, si, si... et moi, j'aurais pu faire la 
même chose, avec quelqu'un d'autre. Je pense que plutôt ça. Après, j'sais pas.

$[\ldots]$

- Et donc les autres, les gens des autres communautés qui pourraient venir... du Maroc, de Turquie ou autre, du Maroc, peut-être, y en avait sans doute dans...

- Les gens qui nous ont défendus ?

- Oui.

- Y avait plutôt beaucoup, Portugais, y avait aussi des Français aussi, mais en fait je pense oui, y avait que des Français et Portugais, quoi. Dans là où on était, y avait que ça. Y avait, après... après, j'sais pas, non y avait que Portugais et Français, y avait que ça.

$-O k$.

- Parce qu'on avait, on avait un gars, là, qui nous a aidés, un petit peu, oui, même un peu beaucoup, il nous a aidés... et puis Alexandra [avec qui Chaban a maintenant deux enfants], qui nous a aidés beaucoup. Et après, dès qu'il y avait un souci, par exemple, on avait des gens là-haut qui pouvaient avertir tout le monde, quoi.

- Ah oui...

- Après y avait des gens aussi de la ville qui nous ont aidés. Ils sont venus, Dominique Grador, Sylvie Christophe (deux élues municipales], Y avait Erwin...

- Bliesenick...

- Bliesenick, voila c'est ça, avec sa femme, y avait Gilles... Masson, Massault [tous militants du collectif RESF], un truc comme ça, y avait lui qui nous a aidés beaucoup, après y avait la presse qui sont venus et nous a posé des questions, voilà, quoi.

\section{Clé $n^{\circ} 5$ : l'apprentissage de la langue et la scolarisation.}

- Et après, quand on est arrivés là, du coup je parlais un peu anglais et on arrivait un peu à se comprendre, quoi, parler anglais un petit peu, et voilà, quoi. Et après, ils m'ont appris certains mots, voilà, ça a continué comme ça. Après, les combats de foot, après, je commençais à faire un peu de foot et c'est là que j'ai commencé un peu [à apprendre le français].

$[\ldots]$

- Et oui, donc alors le fait d'arriver ici, dans ces conditions, y avait, par rapport à tout ce qu'il y a pu avoir comme conflits avec la police, ou comme difficultés à vivre, c'est là que vous avez trouvé de l'appui, avec les voisins... Comment ? Tu dirais que ça c'est passé comment?

- Ben au départ, c'était un peu dur, un peu dur pour se comprendre avec les voisins. A 
l'époque, quand on est arrivés, moi je parlais un peu anglais, avec... parce que j'ai appris un peu avec des amis là-bas en Hongrie... que j'avais déjà connus en Serbie. Et après, quand on est arrivés là, du coup je parlais un peu anglais et on arrivait un peu à se comprendre, quoi, parler anglais un petit peu, et voilà, quoi. Et après, ils m'ont appris certains mots, voilà, ça a continué comme ça.

- Et donc toi, c'est dans cette période là que tu as commencé l'école, tu t'es inscrit pour le $C A P$, ton premier CAP?

- En fait, quand je suis arrivé en France, j'ai expliqué que j'ai que trois mois d'école, dans mon pays. Je savais pas... je savais lire, je savais écrire, mais tout qu'est-ce que je savais, en fait, j'ai appris par la télé, en fait en regardant la télé, parce que chez nous ça passe beaucoup des séries espagnoles et tout ça, avec version originale.

- Ah oui, en sous-titré.

- En sous-titré, voilà. Du coup, c'est comme ça, on est... pas que moi, y a du monde qu'est comme ça, on apprend. Et voilà. Et quand je suis arrivé en France, ils m'ont mis directement au lycée. Au lycée, moi j'étais paumé, car j'avais pas le niveau, je connaissais pas vraiment la langue, je parlais pas beaucoup, et je savais pas ni écrire, ni écrire ni lire [en français]. C'est tout qu'est ce que je savais, certains trucs, en anglais, que je parlais avec un gars à Dijon, là, on était dans un truc... en fait pour les SDF, où ils dormaient, et ils dormaient, que des fois ils venaient manger dans un endroit où tous les midis, ben on allait manger là-bas. Et du coup, c'est là-bas que j'ai appris un petit peu, et il m'a dit... moi, je parlais en anglais, lui en français, et du coup moi je notais. Je notais, en fait, avec les lettres que je...

- Oui, phonétiquement, oui.

- C'est ça. Voilà, avec des... en abrégé, quoi. Comme en serbe, quoi. Voilà, et du coup c'est comme ça que je commençais un petit peu... certains mots, mais pas tout. Quand je suis arrivé au lycée, c'était trop trop dur. Après, ils m'ont transféré, ils m'ont transféré dans une ... dans une petite école pour les gens qui ont des difficultés en français, en math et tout ça. C'était... j'étais six mois là-bas, c'est là que j'ai appris bien français, avec Madame Jannie... je me rappelle plus sons nom. Et voilà, quoi. Et puis et puis j'ai commencé faire après mon CAP, je l'ai obtenu. J'ai un CAP de menuiserie fabriquant, j'ai fait mes stages dans une entreprise, et après j'ai fait un autre CAP comme installateur, et j'ai fait tout mes stages, là, tous mes CAP, j'ai fait dans la même entreprise et du coup ils m'ont pris pour six mois, un contrat de six mois. Et après y avait pas de travail, du coup il a pas pu me renouveler encore plus, et du coup, maintenant, voilà, quoi. 


\section{Clé $n^{\circ} 6$ : les premiers emplois.}

- Donc ce passage, on va dire par l'école, quoi... par l'apprentissage, où tu avais cette alternance entre l'école et l'entreprise, ça c'est quelque chose qui, pour toi, a permis d'accélérer ou de faciliter l'intégration?

- Depuis là-bas ?

- Oui. Et ici, aussi, par rapport à quand vous êtes arrivés tous ensemble, est-ce que tu ... parce que tu es arrivé, toi, jeune majeur et en étant dans cette situation d'apprentissage, est-ce que ça a rendu les choses plus faciles pour l'intégration pour toi, ou pas ?

- Oui, bien sûr ! Non mais bien sûr ! Parce que déjà... déjà je voyais les gens... comment dire ? Je voyais déjà leur culture, déjà ils m'ont appris plein de choses, et déjà j'avais un boulot et tout. Oui, oui, déjà je sais, ça change beaucoup de choses pour moi.

- Tu as aussi travaillé... parce que tu as pas travaillé qu'à Tulle, là tu es en ce moment sur Argentat.

- Oui, à Blocfer.

- Et auparavant, tu as été dans d'autres petites communes...

- Oui, oui. J'étais chez... mon premier boulot, c'était chez Rioux [l'entreprise de menuiserie où Chaban Tatari a fait ses CAP puis son premier contrat de travail].

- Oui, oui.

- Mon deuxième boulot, c'était à Smurfit-Kappa, c'était à Uzerche, c'était dans une usine.

- À Smurfit, oui.

- Mais c'était pas le même métier, c'était... on fabriquait des cartons. Heu... après j'allais dans des petites entreprises en menuiserie, un peu fabriquant, un peu installateur. Voilà, et du coup, mon dernier métier, là, c'est, heu... à Blocfer, quoi. Et actuellement, ça se passe très bien, et... voilà. [...] Mais en fait, j'ai aussi des cousins, là, qui commençaient à aller à l'école, qui sont au collège, et y en a certains qui ont trouvé du boulot déjà, y en a un qui travaille avec un contrat aidé, mais qui est en CDI. Et y en a un autre cousin qui a fini son apprentissage, mais qui en ce moment qu'il embauche pour du travail. Et j'ai un autre cousin, là, lui qui est carrossier, je pense, oui c'est ça, et il fait les études aussi. Et il est en CAP, hé bien lui, c'est deux lycées différents, il a un lycée à Limoges, et y a un lycée à Brive ; du coup... Après, le petit cousin, là, Ferdi, il est probablement au collège avec l'autre... Après, Rosalinda, la grande cousine, elle est probablement en apprentissage [après un apprentissage à Treignac, elle a trouvé un emploi dans un supermarché]. 


\section{Clé $n^{\circ} 7$ : l'accueil dans l'arrondissement de Tulle, le respect dans la ruralité.}

- Tulle, c'est quand même une petite ville ; tu es à Uzerche ou à Argentat aussi, c'est des toutes petites villes... où qu'on soit, on voit la campagne autour... Et est-ce que tu penses que le fait que tu sois dans un endroit comme ça, très rural, très campagne, ça change quelque chose ? Pour toi, d'une part, et puis pour la famille, par rapport à si vous étiez en ville, dans une grande ville?

- Ben déjà, la tranquillité, déjà. Déjà, les gens, ils sont plus respectueux... Et en ville, aussi, ils ont peur, quoi. Parce que là-bas... Belgrade, c'est pas une petite ville, c'est une grande ville, et nous on habitait à l'extérieur de Belgrade. Mais du coup, c'était... même si c'était un petit village, mais quand même, c'était...

- C'était la banlieue, quand même...

- Voilà, c'est ça. Y avait des gens, qu'on avait juste à côté, qui sont partis du Kosovo, parce que par rapport à la guerre ils sont venus en Serbie, du coup, à chaque fois, on avait des problèmes avec eux, et ils venaient la nuit, et ils jetaient des cailloux, et tout ça. Du coup, le truc que ça change par rapport à là-bas, c'est là on est tranquilles. Là on est avec des gens qui sont respectueux, qui voient pas la différence... enfin ils voient pas la différence de peau, ils voient pas la différence de nationalité, ni... ni l'islam, ni rien du tout. Quoi ? Si, si... ils ont... comment dire ? Ils ont rien à faire si tu es un musulman ou chrétien ou orthodoxe ou je sais pas... II faut juste que tu sois un humain, respectueux, que tu respectes. Si... si je les respecte, ils vont me respecter, mais si je respecte pas, c'est normal qu'ils vont pas me respecter. Voilà, que ça change que là on est tranquille, quoi. Avec des gens qui sont formidables.

$[\ldots]$

- Parce que en France, on a une image, aujourd'hui, qui est transmise, avec le fait qu'on en parle beaucoup à la télé, dans les médias, quoi, et à côté de ça, c'est peut-être une image d'une certaine France... en ville ou dans certaines banlieues, qui correspondrait pas du tout à ce qui peut se vivre ici, dans ce milieu plus rural.

- Mmm. Non, non. Et non, là, on se sent pas être menacés. Pas comme là-bas, quoi. Là c'est... on est libre, là comme je le disais tout à l'heure.

\section{Clé $n^{\circ} 8$ : comparer avec une banlieue (Berlin).}

- Mais ça c'est des choses dont vous avez pas parlé... ? Quand tu as revu ta famille, tes parents... 
- En Allemagne... [...] Mais... je pense que là-bas, c'est un peu, un peu... ça dépend des gens aussi, là-bas, aussi, parce que y a des gens aussi qui sont sympas, et y a des gens aussi qui... qui ont du mal à accepter... les étrangers.

- Oui.

- Voilà. Et du coup, là-bas en Allemagne, c'est... c'est un peu...

- C'est plus tendu, hein?

- Oui, c'est plus tendu, c'est pas comme en France.

- Et puis y avait... y a des agressions...

- C'est ça.

- Contre les gens, contre les foyers d'accueil...

- C'est ça, oui.

- Vous en avez parlé un peu, de ces choses-là, avec la famille?

- Non, non, ils m'ont jamais... Parce que, en fait, c'est la famille et ils restent vraiment entre eux et du coup... c'est pas comme ici où moi j'ai eu la chance, hein, d'avoir des amis et de découvrir un peu leur culture... y a énormément de vie, que j'ai actuellement comme eux, quoi, comme les Français. Et du coup, du coup, là-bas, ils ont pas la chance, ils ont pas la chance d'avoir ça.

- Et aussi ils sont dans des grandes villes.

- C'est ça.

- ... dans lesquelles on va se retrouver dans des banlieues ou dans des HLM dans lesquels y a pas forcément, peut-être, de ... beaucoup d'Allemands.

- Mais... voilà. Voilà, c'est ça. Surtout, quand y en a... par exemple quand il vient des étrangers, ils vivent carrément ensemble, avec des étrangers, mais pas avec les Allemands. Et dans des quartiers... et ben après, sûrement, peut-être. Voilà. Moi je connais pas vraiment là-bas, ça fait quatre-cinq fois que je vais là-bas, et du coup j'y vais surtout pour voir ma famille, et du coup je sors pas vraiment. Si... j'ai... de temps en temps en ville, mais pas beaucoup, quoi, et là où par exemple, là où mes parents où ils habitent, y a beaucoup des étrangers. Après, y a, y a des Allemands, c'est plutôt vers un peu... un peu extérieur, quoi. En fait, ils ont, comment dire, ils ont une entrée, et...

- Oui. Ça peut être la même commune, mais pas exactement, l'autre bout du quartier...

- Ben voilà. C'est ça, c'est ça. Et du coup, voilà, quoi. C'est pas comme ici, quoi. Ça, par exemple, y a... Si par exemple un étranger vient, ils vont le mettre, là par exemple, si y a un Français en face, ils vont le mettre, quoi. Et là-bas, je pense pas que c'est comme ça, quoi. Après, c'est peut-être, sûrement... je connais pas. 


\section{Clé n 9 : « Liberté, égalité, fraternité », devenir Français.}

- Et quand vous vivez tout ça et que bon, vous arrivez, donc, un a un peu parlé tout à I'heure que vous maintenez, vous, par rapport à la langue, par rapport à des coutumes..., mais finalement par ailleurs, même si la question est pas simple par rapport aux papiers, petit à petit, je vois bien que tu es en train, ben tu deviens Français, quoi. Comment tu vis ça, toi ?

- Mais, finalement c'est... comment expliquer ? Ben, déjà, j'ai ma fille qu'elle est française, et je vis aussi avec ma femme qu'est française aussi. Du coup, ça change beaucoup de chose. Du coup, je m'intègre à... à la culture française. Et puis, et puis c'est bien, quoi. Après, je sais pas comment expliquer.

- Est-ce que tu te sens plutôt, on va dire... il peut y avoir plusieurs réponses, mais est-ce que tu te sens toujours, finalement, appartenir à la communauté Rom ou Ashkali originaire du Kosovo, est-ce que tu te sens plutôt appartenir à ce pays de Tulle, au sens large ?

- Moi, qu'est ce que j'aimerais...

-Oui?

- Moi, qu'est ce que j'aimerais... J'aimerais avoir la nationalité français. Parce que je peux le dire que j'aime pas trop mon pays, parce que là-bas les gens, ils veulent pas trop m'accepter, tel que je suis, et là ils m'acceptent. Et le problème c'est quoi ? J'ai pas droit à la nationalité français, parce que j'ai pas l'acte de naissance, et du coup, surtout que ça bloque tout, et voilà, quoi. Du coup, moi, j'aimerais bien, j'aimerais bien même qu'avoir la nationalité français et après, moi je me sens quoi, je me sens... un peu comme chez moi, quoi.

- Alors aujourd'hui, dans le débat politique en France, y a beaucoup de choses qui reviennent sur les notions de valeurs. Que tu sais, la devise de la République, Liberté, égalité, fraternité. [...] Et toi, tu le vis comment, le rapport à toutes ces valeurs, ces idées communes qui font, ben, qu'on se sent être français ?

- Mon essai était déjà de s'intégrer, avec les français, et après, soit... Ah, j'ai du mal à expliquer, je sais pas comment expliquer, quoi.

- Peut-être que ma question est pas très simple. Elle est trop compliquée, sans doute, y a trop de mots dedans.

$-\mathrm{Mmm}$ !

- Mais, par rapport à ce que tu dis, que vous avez pu vivre avec le regard particulier des Serbes par rapport à vous, et puis pareil en Hongrie, quand t'arrives ici, et que ici, finalement, on écrit sur le fronton du tribunal, Liberté égalité fraternité, j'ai un peu 
l'impression que c'est quand même des choses qui...

- Oui. Ben oui, d'ailleurs. Parce comme je disais tout à l'heure, là-bas les gens m'acceptaient pas, quoi, mais là, ils m'acceptent. Parce que c'est un pays libre, et les gens ils sont pas pareils, quoi. Ils sont pas pareils du tout, et du coup, tu vis... là je vis sans peur, quoi. Là, je sors droit, même la nuit et tout, j'ai aucune peur, quoi.

- Mais tu dis, oui, alors, mais le fait qu'il pouvait y avoir, comme ça, une part d'agressivité et des risques à sortir, c'était lié au fait que vous soyez Roms, ou que soyez pas du pays? - Ben déjà, la couleur de peau, différent.

- Ouais.

- Par exemple, même si c'est un musulman, si c'est... il est blanc, on peut pas savoir si c'est un musulman, ou un Serbe, ou un truc... Moi... Qu'il est blanc, qu'il ait la même couleur de peau, quoi, c'est ça qui compte. Et là-bas, c'est tu vois que tu as une couleur de peau différente, ils vont te taper, et voilà. Mais là, c'est le sort, par exemple là, n'importe quelle heure, une nuit, minuit ou une heure du matin, voire un truc comme ça, je sais que j'aurais aucun souci. Que ça soit là ou que ce soit dans une grande ville, à mon avis, tu vois quoi c'est pas pareil comme là-bas. C'est ça qu'est la différence de là-bas, quoi.

- Oui.

- Là, on se sent un peu plus libre, quoi.

- Oui.

- Et du coup, c'est ça que c'est, que ça fait... que ça fait aimer à rester là, quoi, et se sentir un peu comme chez soi. Que... qu'est ce qu'on a pas pu avoir là-bas. J'sais pas si tu comprends qu'est-ce que je dis, parce que j'ai du mal, j'ai du mal à répondre, mais...

\section{Clé $\mathrm{n}^{\circ} 10$ : la tolérance interreligieuse.}

- Je pense, par rapport à tout à l'heure, quand on mangeait, et on a parlé un peu de religion. Donc, en fait, toi t'as deux filles. .

- Oui.

- Et finalement, l'aînée, vous parlez pas particulièrement, ou j'avais l'impression... que y avait pas forcément d'approche de la...

- J'ai pas compris... j'ai pas compris le début...

- Vous parlez pas particulièrement de religion, avec l'aînée ?

- Ah ! Non non non non.

- Voilà. Mais y a quelques trucs sur l'alimentation, des trucs comme ça ?

- Ben après, si tu veux, ma femme, elle est française, mais moi je suis... vu qu'elle est 
catholique, et moi je suis musulman... après c'est pas moi qui a demandé, comment dire, de... de, enfin, de faire sans porc ou des trucs comme ça, voila. C'est elle qui l'a voulu. Par exemple, de ne pas boire ni alcool, ni du porc à la maison, ben c'est elle qui l'a décidé, et après, pour ma fille, moi je dis, c'est à elle de décider, si elle voudra, par exemple, manger du porc, elle mangera, si elle voudra. Si elle voudra pas, ben voila, y a personne, personne qui va la forcer. Qu'est-ce qu'on parlait tout à l'heure, on est dans un pays libre. Du coup, c'est à eux de décider, c'est pas à nous de décider.

- Oui.

- Et du coup, voila, c'est, c'est elle qui l'a dit ça, et du coup, alors après...

- Mais Alexandra, vis-à-vis de toi, quand elle fait ça... quand elle propose ça aussi par rapport aux filles, c'est qu'elle est aussi, sans doute, attachée à qui tu es, et à ce que tu conserves ce... cette culture, ces éléments de ton origine, tes origines.

- Mais... ben je pense que c'est ça, oui, même c'est certain, parce qu'elle est... Elle me respecte, et moi j'essaie de respecter... par exemple, chez nous, les Nouvel an, les Noël, on le fait pas, et moi je le fait, parce que... parce que quand c'est Ramadan, elle le fait, moi je lui ai jamais rien demandé, et du coup, elle voulait, toute seule, faire Ramadan. On l'a fait ensemble, ça fait plusieurs années qu'on le fait ensemble. Et du coup, quand c'est Noël, on le fait ensemble aussi, parce que même si c'est pas mon truc à moi, à nous, quoi, les musulmans, moi je le fait pour elle, parce je respecte, parce que je me dis... parce qu'elle fait Ramadan, mais pourquoi moi, pourquoi je peux pas faire Noël ? Du coup, on le fait en famille, quoi. Et voila, et du coup, on essaye de se faire respecter, quoi. De... de se... de ne pas... comment dire, que je lui interdis pas, par exemple, ses habitude qu'elle a, elle a pour tous les jours.

\section{Conclusion.}

Que valent les dix clés qui ont été repérées, et peut-on les connecter entre elles ?

Pour ce qui est de questionner des appariements, on a constaté au fil de l'entretien avec Chaban que son souhait de devenir Français n'est que très indirectement une des conséquences d'une purification ethnique dans les Balkans. Le résultat immédiat de cette politique raciste est d'avoir jeté des communautés dans le parcours semé d'embûches de l'immigration dans l'UE. Ce qui a permis une intégration relativement satisfaisante de ce groupe de Roms et d'Ashkalis, ce sont des facteurs humanistes comme la proposition d'insertion par le sport et l'appui fourni par les voisins et les aidants dans un contexte favorable d'accueil et de respect au sein d'un territoire marqué par la ruralité, et des facteurs socio-institutionnels comme l'apprentissage de la langue et la scolarisation, 
facilitant les uns comme les autres l'accès aux premiers emplois. Le tout fut accompagné d'une démarche réflexive personnelle de Chaban et de ses compagnons sur divers aspects de la vie sociale dans le coin de Corrèze où ils sont arrivés, notamment en prenant en compte la comparaison avec la vie de parents dans banlieue de Berlin et l'expérience de la tolérance interreligieuse.

Quant à la validité des clés identifiées et des éléments d'analyse proposés sur la foi des propos enregistrés, elle demanderait maintenant à être vérifiée en poussant plus avant la mise en œuvre d'une anthropologie de l'ordinaire ${ }^{3}$ en réalisant d'autres entretiens avec au minimum un.e autre membre de ce groupe d'immigrés dont l'« appartenir » est encore en devenir, et avec des acteurs locaux qui ont pu les accompagner.

Didier CHRISTOPHE, ENSFEA.

3 Cf. Éric Chauvier (2011), Anthropologie de l'ordinaire. Une conversion du regard, Toulouse, Anacharsis. 